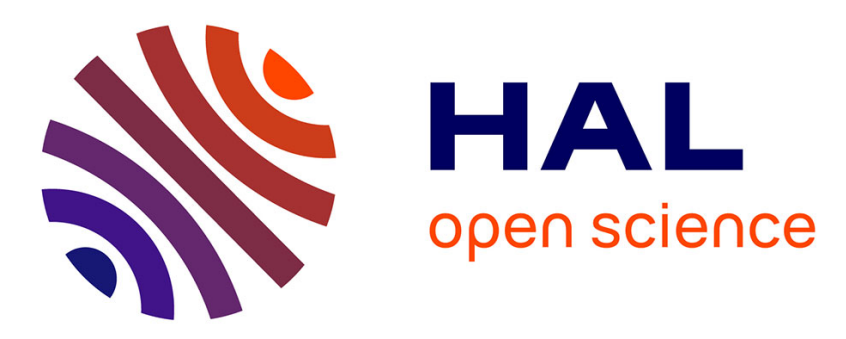

\title{
Ranking of the nonlinearities of electrodynamic loudspeakers
}

Romain Ravaud, Guy Lemarquand, T. Roussel, Valérie Lemarquand

\section{To cite this version:}

Romain Ravaud, Guy Lemarquand, T. Roussel, Valérie Lemarquand. Ranking of the nonlinearities of electrodynamic loudspeakers. Archives of Acoustics, 2010, 35 (1), pp.49-66. hal-00438763

\section{HAL Id: hal-00438763 https://hal.science/hal-00438763}

Submitted on 4 Dec 2009

HAL is a multi-disciplinary open access archive for the deposit and dissemination of scientific research documents, whether they are published or not. The documents may come from teaching and research institutions in France or abroad, or from public or private research centers.
L'archive ouverte pluridisciplinaire HAL, est destinée au dépôt et à la diffusion de documents scientifiques de niveau recherche, publiés ou non, émanant des établissements d'enseignement et de recherche français ou étrangers, des laboratoires publics ou privés. 


\title{
Ranking of the nonlinearities of electrodynamic loudspeakers
}

\author{
December 3, 2009
}

\section{rameters, nonlinear modeling}

\footnotetext{
${ }^{1}$ Corresponding author : guy.lemarquand@ieee.org
} The authors are with the Laboratoire d'Acoustique de l'Universite du Maine, UMR CNRS 6613, Avenue Olivier Messiaen, 72085 Le Mans Cedex 9, France 


\section{${ }_{18} 1$ INTRODUCTION} 36 [20]-[22]. [11]-[16].

The reference model describing the electrodynamic loudspeaker was designed by Thiele and Small [1]. Their model was adapted to describe an electrodynamic loudspeaker as a linear system. This model is very useful because it is very simple to use and its parameters can be conveniently presented in terms of an electric analog circuit [2]. However, a loudspeaker shows nonlinearities that produce distortions. These nonlinearities have three major sources [3][4]: the suspensions, the diaphragm [5]-[7] and the motor [8]-[10]. They have been largely studied and there are many attempts to model them

However, most of the papers dealing with the nonlinearities of electrodynamic loudspeakers do not take into account the variations of the mechanical damping $R_{m s}$ or the equivalent mass $M_{m s}$. But these parameters are also nonlinear and their variations must be taken into account in order to precisely characterize the distortions created by electrodynamic loudspeakers. It can be noted that alternative loudspeakers structure without iron have been proposed and studied [17]-[19] which use ferrofluid suspensions in order to delete the classical distortions generated by electrodynamic loudspeakers

This paper has two objectives. In the first part, we present an experimental method which allows us to determine precisely the variations of the small signal parameters according to the input current. Our experimental method is based on a very precise measurement of the electrical impedance of 
the electrodynamic loudspeaker. Indeed, the measurement accuracy is about $10^{-4} \mathrm{Ohm}$ for both the real and imaginary parts of the electrical impedance. However, all our approach does not use the classical definition of an electrical impedance. An electrical impedance, commonly defined by its ratio $\frac{U}{I}$ (in the frequency domain) does not depend on input current. Indeed, this electrical impedance is only defined for linear systems.

However, our experimental measurement shows that this ratio $\frac{U}{I}$ depends on $I$. This can be easily verified experimentally by measuring the experimental impedance for different input currents. Such a result is in fact known for scientifics involved in the modeling of nonlinear systems: a nonlinear system depends generally on the input level. In the case of an electrodynamic loudspeaker, the electrical impedance, that is to say, the ratio $\frac{U}{I}$ depends on the input level.

54 We also show that it exists a bijective relation between the input current $I$ and the position of the voice coil $X$. This result is of great importance because it shows that the non linear parameters describing an electrodynamic loudspeaker $\left(B l(x), R_{m s}(x)\right.$ and $\left.L_{e}(x)\right)$ can also be described as parameters depending on input current (in the time-domain or in the frequency-domain).

59 It can be noted that, strictly speaking, the force factor $B l$ which is commonly used in analog circuits represents the mean induction field times the length $l$ of the voice coil inside the air gap. Therefore, when the voice coil position $x(t)$ is sinusoidal, in complex notations, the force factor should be seen as a mean force factor $\tilde{B} l$.

In the second part, we discuss the behaviour of each nonlinearity accord- 
ing to both the input level and the input frequency. It is noted that all this part is treated theoretically. Indeed, a very good agreement between our analytical model and experimental measurements has confirmed that our model can be used for modelling the distortions created by an electrodynamic loudspeaker. We show here that the lumped parameter whose relative variation according to input current is the most important is not always the same and depends greatly on both the input level and the input frequency.

Another drawback known in electrodynamic loudspeakers is that it is a time-varying system. In fact, the electrical resistance increases with time and the mechanical compliance of the outer rims depends also on time. These dependence have been studied with a similar approach in previous papers. In this paper, some care has been taken in the experimental measurements for omitting these temporal effects.

This paper proposes a way of ranking the nonlinearities of electrodynamic loudspeakers. To our knowledge, this way of characterizing these nonlinearties has never been used for electrodynamic loudspeakers. However, many new phenomena can be seen by using such an experimental approach. This is why such results are very interesting for many manufacturers involved in the design of electrodynamic loudspeakers. We precise here that we use for the rest of this paper the small notations $x(t)$ and $i(t)$, the displacement of the voice coil and the input current in the voice coil in the time-domain and the capital letters $(X, I, U)$ in the frequency domain. We also precise that $U$ is the input voltage. 


\section{Classical description of a loudspeaker and its limits}

\subsection{The small signal model using lumped parameters}

A common way of characterizing an electrodynamic loudspeaker is to measure its electrical impedance $Z e$. Its theoretical expression is well known and is given by Eq. (1).

$$
Z_{e}=R_{e}+\frac{j L_{e} w R_{\mu}}{j L_{e} w+R_{\mu}}+\frac{B l^{2}}{R_{m s}+j M_{m s} w+\frac{1}{j C_{m s} w}}
$$

where all the parameters are defined below.

$B l=$ electrodynamic driving parameter $[T . m]$

$R_{m s}=$ mechanical damping, drag force $\left[N . s . m^{-1}\right]$

$C_{m s}=$ mechanical compliance of suspension( spider, outer rim) $\left[N^{-1} . m\right]$

$L_{e}=$ inductance of voice coil $[H]$

$M_{m s}=$ equivalent mass of moving voice coil, cone and air $[\mathrm{kg}]$

$R e=$ electrical resistance of voice coil $[\Omega]$

$R \mu=$ eddy current resistance $[\Omega]$

$w=$ angular frequency $\left[\mathrm{rad} . \mathrm{s}^{-1}\right]$

These parameters can be represented in terms of an analog circuit in Fig. 1.

According to (1), the electrical impedance $Z_{e}$ does not depend on $I$. However, an experimental measurement shows the contrary. Indeed, the real part and the imaginary part of this electrical impedance are represented by using the Nyquist diagram in Fig 2 and we see that this electrical impedance de- 

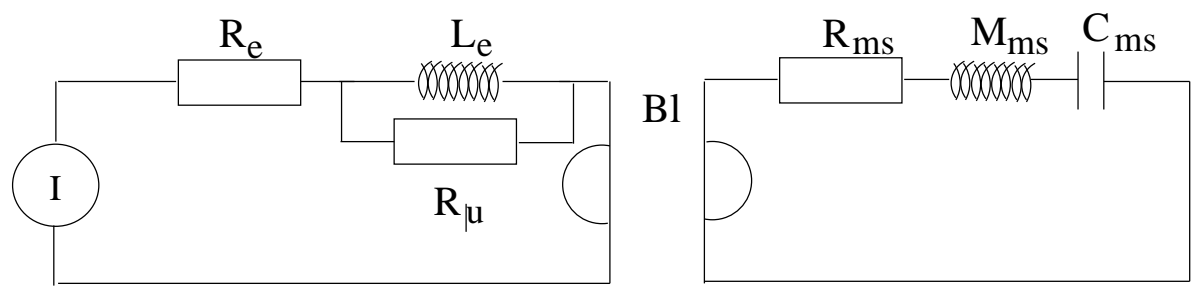

Figure 1: Representation of the lumped parameters in terms of an analog circuit

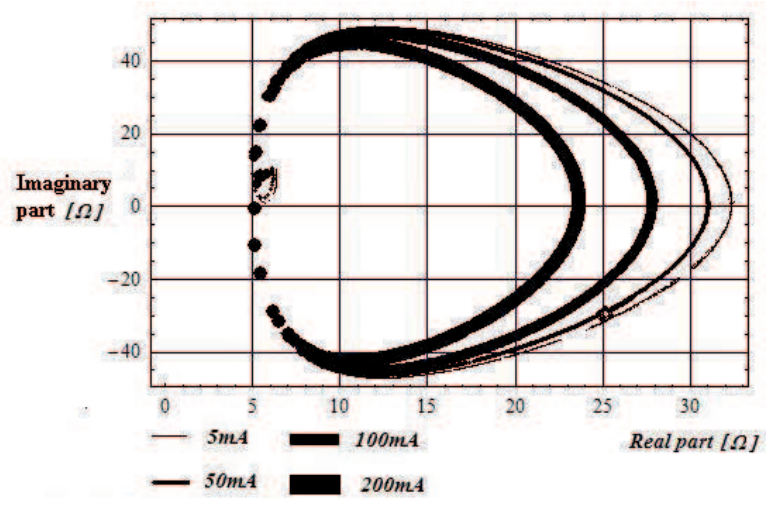

Figure 2: Experimental representation in the complex plane of the electrical impedance of the electrodynamic loudspeaker for four coil current levels ( $5 \mathrm{~mA}, 50 \mathrm{~mA}, 100 \mathrm{~mA}, 200 \mathrm{~mA}$ )

pends on the input current. Consequently, this eletrical impedance can be

seen as a nonlinear electrical impedance. We denote it $Z_{e}^{(N L)}(I)$. In short, we can say that the classical electrical impedance $Z_{e}$ characterizing an electrodynamic loudspeaker is not constant when the input current varies. Consequently, we use the notation $Z_{e}^{(N L)}(I)$ to describe the electrical impedance. However, strictly speaking, the term -electrical impedance- should not be used to describe a nonlinear system like an electrodynamic loudspeaker.

As a consequence, the lumped parameters defined in Eq. (1) depend on 
the input current. The main problem is thus to know how to characterize their dependence according to the input current. According to the current state of art, cited in the introduction of this paper, the dominant nonlinearities in electrodynamic loudspeakers are $B l(x), C_{m s}(x)$ and $L_{e}(x)$. However, it exists a relation between the voice coil position $x$ and the input current $i$. This relation is shown in next section. Consequently, it can be noted that these nonlinearities can depend on $x$ or $i$ : it is in fact equivalent. In this paper, we choose to work directly with the input current $i$ because $Z_{e}^{(N L)}(I)$ is easier to determine experimentally. Consequently, thanks to this very precise experimental measurement, the variations of the lumped parameters can be determined according to the input current $i$.

\subsection{Relation between the input current $I$ and the voice coil position $X$ according to the Thiele and Small model}

This section presents the relation between the input curreent $I$ and the voice coil position $X$ with the linear approximation of the Thiele and Small model.

It is noted here that the aim of this section is merely to show that it exists a bijective relation between the input current I and the voice coil position X. To find this relation, we use one differential equation describing the electrodynamic loudspeaker and we use complex notations. We find:

$$
\left(M_{m s}(j w)^{2}+R_{m s}(j w)+\frac{1}{C_{m s}}\right) X=B l I
$$

So, we can write: 


$$
X=\frac{B l}{\left(M_{m s}(j w)^{2}+R_{m s}(j w)+\frac{1}{C_{m s}}\right)} I
$$

Therefore, we see that Eq. (3) is the bijective relation between the input current $I$ and the voice coil position $X$, in complex notation. This relation shows that we can work either with the input current $I$ or with the voice coil position $X$ so as to describe the nonlinear variations of the Thiele and Small parameters.

\subsection{Relation between the input current $I$ and the voice coil position $X$ according to the nonlinear model used in this paper}

Strictly speaking, the relation between the input current I and the voice coil position is nonlinear. Indeed, all the small signal parameters depend on the input current. It is emphasized here that the transfer function describing the ratio $\frac{U}{I}$ is considered as the ratio of the part of response with fundamental frequency $w$ to the excitation. We call it a nonlinear electric impedance in this paper but it is useful to precise that nonlinear systems cannot be described with complex notations.

We describe in the next section a way of determining the nonlinear laws of all the small signal parameters. Therefore, we precise here for the rest of this paper that (3) can be written in the frequency-domain as follows(4):

$$
X=\frac{B l(I)}{\left(M_{m s}(j w)(I)^{2}+R_{m s}(I)(j w)+\frac{1}{C_{m s}(I)}\right)} I
$$


It is noted that the determination of the small signal parameters can be determined in the frequency domain. We explain in next section how to find these variations.

\section{Determination of the nonlinear variations of the lumped parameters}

\subsection{Principle of the measurement}

We have shown in the previous section that the nonlinear variations of the lumped parameters describing an electrodynamic loudspeaker depend on the input current. We explain now how to find these variations.

The electrodynamic loudspeaker used is a boomer (mark: Eminence, number: beta 15 ). We use the electrical impedance of the electrodynamic loudspeaker because its measurement is very precise. Our measurement device is a Wayne Kerr bridge which has an excellent precision $\left(10^{-4} \Omega\right)$. This experimental devices is dedicated to the impedance measurement and functions as a classical impedance bridge.

In order to measure the electrical impedance of a loudspeaker, it is mounted in a normalized plane in an anechoic room [23]. Dalmont [24] has shown that the acoustical impedance in these conditions is the same as the one when the loudspeaker is mounted in an infinite baffle in an anechoic room. We measure the electrical impedance by varying the frequency and the coil current. So, we build an experimental impedance layer by using a Runge Kutta algorithm to determine all the experimental measured points. In other words, this measurement algorithm chooses the best measurement point according 
to the gradient method. Such a measurement method allows us to detect all the subtle effects due to the nonlinearities in electrodynamic loudspeakers. This algorithm has been improved in relation to the one used in a previous paper. In the previous paper, the algorithm took some experimental points by using constant intensities. In other words, our measurement system used the algorithm of gradient only in two dimensions for one intensity and then it was repeated for another intensity. For example, an intensity was fixed (for example $50 \mathrm{~mA}$ ), and a two-dimensional algortihm allowed us to determine the measurement points, that is to say, our algorithm automatically settled one intensity and took some experimental points by using a method based on the gradient method. In this paper, the algorithm uses a Runge Kutta algorithm in three dimensions $\left(Z_{e}, I, f\right)$ and we can rapidly obtain an impedance layer which is very precise. Consequently, the temporal effects can be neglected. A two-dimensional representation of the electrical impedance is shown in Fig. 2: the imaginary part is a function of the real part ( it is Nyquist diagram for different coil currents). We can say that the Wayne Kerr bridge cannot supply currents greater than 0.2A. Consequently, the parameter variations are determined in this current interval.

Figure 3 confirms that the electrical impedance is a function of the input current.

\subsection{Determination of the nonlinear electrical impedance}

The next step is thus to determine the dependence of the small signal parameters with the coil current so as to construct the nonlinear transfer function. 


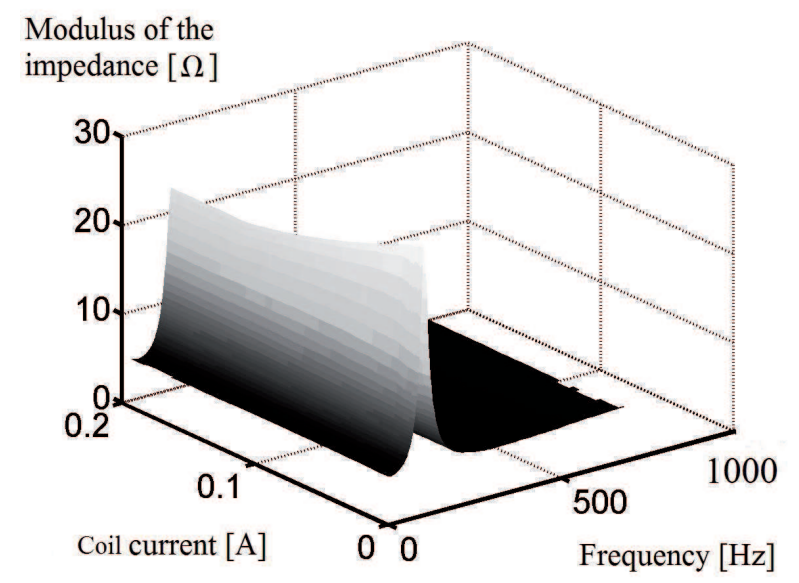

Figure 3: Experimental three-dimensional representation of the modulus of the electrical impedance of the electrodynamic loudspeaker ( $\mathrm{x}$ : coil current 0 A 0,2 A)(y : frequency $0 \mathrm{~Hz} 1000 \mathrm{~Hz})(\mathrm{z}$ : impedance $0 \Omega 30 \Omega$ )

For this purpose, we use the Nyquist diagram constructed previously. Five parameters $\left(B l(I), R_{m s}(I), C_{m s}(I), M_{m s}(I), L_{e}(I)\right)$ are assumed to vary with the coil current. Indeed, the electrical resistance does not depend on input current because our experimental measurements have been done with a stabilized temperature (the electrodynamic loudspeaker has been run in during 24 hours before the experiment) and the eddy current resistance does not seem to vary with the input current $I$. In a first approximation, we use a polynomial writing to represent the dependence of the parameters with the coil current. We write:

$$
B l(I)=B l\left(1+\mu_{B l} I+\mu_{B l}^{2} I^{2}+\ldots+\mu_{B l}^{n} I^{n}\right)
$$

$$
R_{m s}(I)=R_{m s}\left(1+\mu_{R_{m s}} I+\mu_{R_{m s}}^{2} I^{2}+\ldots+\mu_{R_{m s}}^{n} I^{n}\right)
$$

$$
C_{m s}(I)=C_{m s}\left(1+\mu_{C_{m s}} I+\mu_{C_{m s}}^{2} I^{2}+\ldots+\mu_{C_{m s}}^{n} I^{n}\right)
$$

$$
M_{m s}(I)=M_{m s}\left(1+\mu_{M_{m s}} I+\mu_{M_{m s}}^{2} I^{2}+\ldots+\mu_{M_{m s}}^{n} I^{n}\right)
$$




$$
L_{e}(i)=L_{e}\left(1+\mu_{L_{e}} I+\mu_{L_{e}}^{2} I^{2}+\ldots+\mu_{L_{e}}^{n} I^{n}\right)
$$

216

$$
\begin{aligned}
Z_{e}(I)= & R_{e}+\frac{\sum_{s=0}^{n} j L_{e} w R_{\mu}\left(\mu_{L_{e}} I\right)^{s}}{R_{\mu}+\sum_{s=0}^{n} j L_{e} w\left(\mu_{L_{e}} I\right)^{s}} \\
& +\frac{\left(\sum_{s=0}^{n} B l\left(\mu_{B l} I\right)^{s}\right)^{2}}{\sum_{s=0}^{n} j M_{m s} w\left(\mu_{M_{m s}} I\right)^{s}+\sum_{s=0}^{n} R_{m s}\left(\mu_{R_{m s}} I\right)^{s}+\frac{1}{\sum_{s=0}^{n} j w C_{m s}\left(\mu_{C m s} I\right)^{s}}}
\end{aligned}
$$

So the electrical impedance becomes :

We use a least square method to identify all the parameters; this method is based on Symplex algorithm. The Simplex method is a systematic procedure which selects the variable that will produce the largest change towards the minimum solution. This algorithm selects the best choice at each iteration without needing information from previous and future iterations. In our case, the principle of this algorithm is to minimize the difference D between the experimental and the theoretical Nyquist diagrams. Consequently, the two parameters which are minimized are the real part Real $(I)$ and the imaginary part $\operatorname{Imag}(I)$ of the electrical impedance defined by eq. (11).

$$
Z_{e}^{(N L)}(I)=\operatorname{Real}(I)+j \operatorname{Imag}(I)
$$

When the algorithm converges, we obtain the values of the parameters of the equations (5), (6), (7), (8) and (9).

\section{Experimental results}

When we take into account the variations of the small signal parameters with the coil current, the mean difference between the experimental and the 


\begin{tabular}{||l|c|r|r||}
\hline Ranking & Parameter & Law of variation $\mathbf{( 1 0 0 ~ H z )}$ & $\mathbf{D}[\Omega](\mathbf{1 0 0} \mathbf{~ H z})$ \\
\hline 1 & $R_{m s}$ & $1.1\left(1+4.09 I-8.36 I^{2}\right)$ & 1.24 \\
\hline 2 & $\mathrm{Bl}$ & $5.5\left(1+0.33 I-1.02 I^{2}\right)$ & 1.67 \\
\hline 3 & $M_{m s}$ & $0.009\left(1+0.56 I-0.22 I^{2}\right)$ & 1.74 \\
\hline 4 & $C_{m s}$ & $0.00013\left(1+2.02 I-9.3 I^{2}\right)$ & 1.86 \\
\hline 5 & $L_{e}$ & $0.0017\left(1-1.68 I+7.58 I^{2}\right)$ & 1.98 \\
\hline
\end{tabular}

Table 1: Ranking of the parameters according to their sensitivity to the least square algorithm

theoretical values is $0,4 \Omega$ whereas the mean difference is $2,0 \Omega$ with Thiele and Small model with constant parameters. We present in table 1 the laws of variations of the five parameters that vary according to the coil current, and we give for each parameter the sensitivity to the least square. We propose a ranking of these parameters based on the criterion D. To get this ranking, we proceed as follows : we write that one parameter $\left(L_{e}, R_{m s}, M_{m s}, B l\right.$ or $C_{m s}$ ) is a function of the coil current. We input the polynomial in the theoretical impedance and we use the least square algorithm to determine at the value of the mean difference between the experimental impedance and the theoretical impedance. We obtain in this case the ranking shown in table 1. Moreover, the value of the eddy current resistance is $2,2 \Omega$ and the value of the electrical resistance is $3,3 \Omega$. The table 2 shows the laws of variations of Thiele and Small parameters. The representations of these variations are given in Figs 4, 5, 6, 7 and 8. 


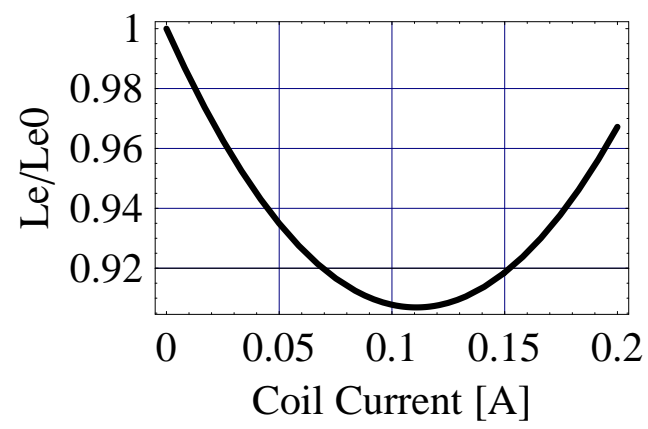

Figure 4: The ratio $\frac{L e}{L e 0}$ is a function of the coil current ( $\mathrm{x}$ : coil current $0 \mathrm{~A}$ $0,2 \mathrm{~A})(\mathrm{f}=100 \mathrm{~Hz}) ; L e 0=0.0017 \mathrm{H}$

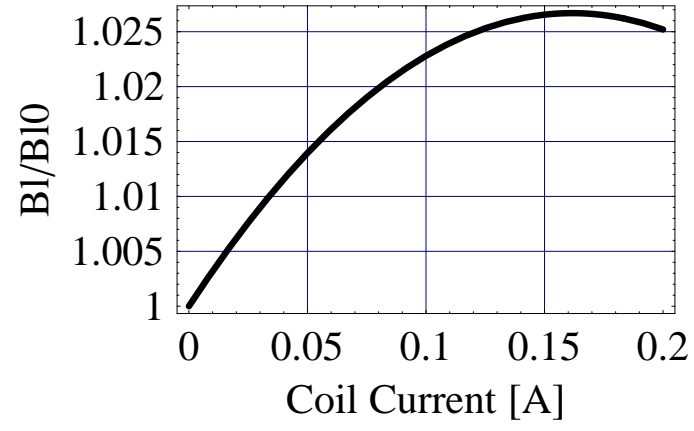

Figure 5: The ratio $\frac{B l}{B l 0}$ is a function of the coil current $(\mathrm{x}$ : coil current $0 \mathrm{~A}$ $0,2 \mathrm{~A})(\mathrm{f}=100 \mathrm{~Hz}) ; B l 0=5.5 T m$

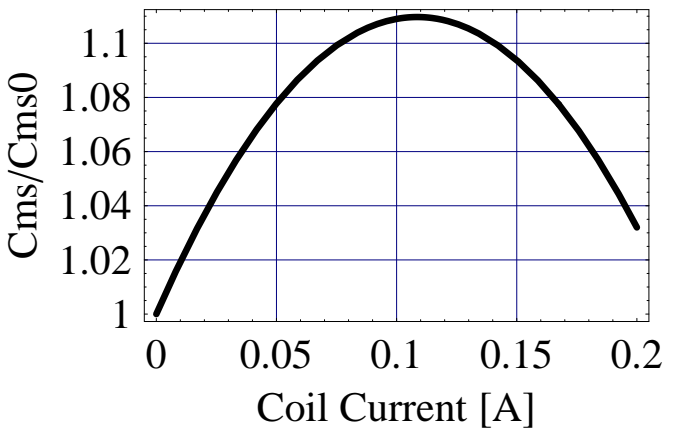

Figure 6: The ratio $C_{m s} / C_{m s 0}$ is a function of the coil current ( x : coil current 0A $0,2 \mathrm{~A})(\mathrm{f}=100 \mathrm{~Hz}) ; C_{m s 0}=0.00013 m / N$ 


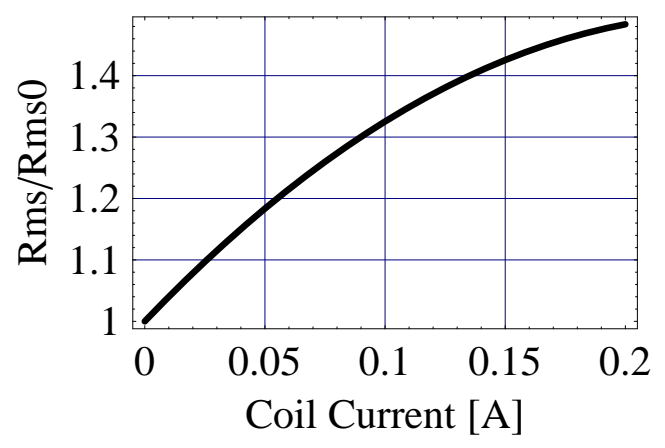

Figure 7: The ratio $\frac{R_{m s}}{R_{m s}}$ is a function of the coil current ( $\mathrm{x}$ : coil current $0 \mathrm{~A} 0,2 \mathrm{~A})(\mathrm{f}=100 \mathrm{~Hz}) ; R_{m s 0}=1.1 \mathrm{~kg} / \mathrm{s}$

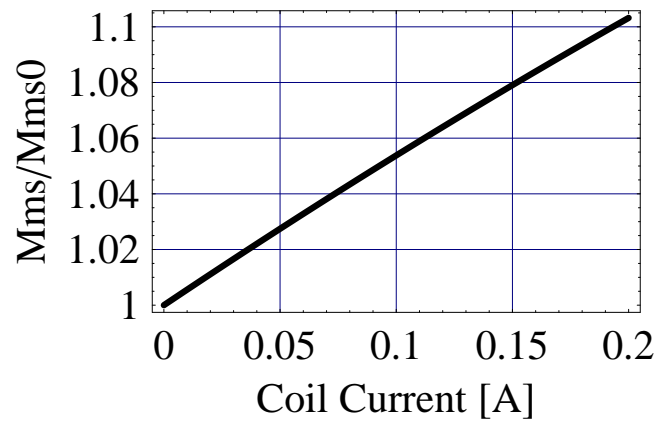

Figure 8: The ratio $\frac{M_{m s}}{M_{m s 0}}$ is a function of the coil current ( $\mathrm{x}$ : coil current $0 \mathrm{~A} 0,2 \mathrm{~A})(\mathrm{f}=100 \mathrm{~Hz}) ; M_{m s 0}^{m s 0}=0.009 \mathrm{~kg}$ 
263

\subsection{Discussion}

The most sensitive parameter to the variations of the coil current is the equivalent damping parameter $R_{m s}$. The parameter which is the less sensitive to the variations of the coil current is the inductance $L_{e}$ of the voice coil. However, though this inductance sensitiveness is rather weak, the relative variation of the inductance $L_{e}$ is rather important. In other words, even if the inductance $L_{e}$ is a parameter that contributes just a little to the variation of its electrical impedance, we see that its relative variation is important. Another interesting point can be adressed. With the values found in this paper, at $200 \mathrm{~Hz}$, we see that the eddy current resistance $R_{\mu}$ equals roughly $w L_{e}$. We can conclude that the effects of the eddy currents become important from $200 \mathrm{~Hz}$ for this electrodynamic loudspeaker.

\section{Study of the distortions created by the elec- trodynamic loudspeaker}

\subsection{Obtaining the nonlinear differential equation}

The nonlinear differential equation describing the electrodynamic loudspeaker is given by Eq. (12). We use the parameter $k=\frac{1}{C_{m s}}$ to solve it.

$$
a(i) \frac{d^{3} x(t)}{d t^{3}}+b(i) \frac{d^{2} x(t)}{d t^{2}}+c(i) \frac{d x(t)}{d t}+d(i) x(t)=u(t)
$$

with

$$
a(i)=\frac{\left(M_{m s}\left(1+\mu_{M_{m s}} i+\mu_{M_{m s}}^{2} i^{2}\right)\right)\left(L e\left(1+\mu_{L e} i+\mu_{L e}^{2} i^{2}\right)\right)}{B l\left(1+\mu_{B l} i+\mu_{B l}^{2} i^{2}\right)}
$$

$$
b(i)=\frac{\left(\left(M_{m s}\left(1+\mu_{M_{m s}} i+\mu_{M_{m s}}^{2} i^{2}\right) R e\right)\right.}{B l\left(1+\mu_{B l} i+\mu_{B l}^{2} i^{2}\right)}
$$


264

$$
+\frac{\left(R_{m s}\left(1+\mu_{R_{m s}} i+\mu_{R_{m s}}^{2} i^{2}\right) R_{e}\right)}{B l\left(1+\mu_{B l} i+\mu_{B l}^{2} i\right)}
$$

$$
\begin{aligned}
c(i)= & \frac{\operatorname{Re}\left(R_{m s}\left(1+\mu_{R_{m s}} i+\mu_{R_{m s}}^{2} i^{2}\right)\right)+\left(B l\left(1+\mu_{B l} i+\mu_{B l}^{2} i^{2}\right)\right)^{2}}{B l\left(1+\mu_{B l} i+\mu_{B l}^{2} i^{2}\right)} \\
& +\frac{\left(L e\left(1+\mu_{L e} i+\mu_{L e}^{2} i^{2}\right)\right) k\left(1+\mu_{k} i+\mu_{k}^{2} i^{2}\right)}{B l\left(1+\mu_{B l} i+\mu_{B l}^{2} i^{2}\right)}
\end{aligned}
$$

$$
d(i)=\frac{R e\left(k\left(1+\mu_{k} i+\mu_{k}^{2} i^{2}\right)\right)}{B l\left(1+\mu_{B l} i+\mu_{B l}^{2} i^{2}\right)}
$$

\subsection{Solving the nonlinear differential equation}

The relation between the coil current $i$ and the position of the voice coil $x$ is nonlinear (Eq. (4)). Each small signal parameter is a function of the voice coil position and we obtain, for example, for one parameter :

$$
R_{m s}(x)=R_{m s}\left(1+\tilde{\mu}_{R_{m s}} x+\tilde{\mu}_{R_{m s}}^{2} x^{2}\right)
$$

with $\left|\tilde{\mu}_{R_{m s}}^{2}\right|<<\left|\tilde{\mu}_{R_{m s}}\right|<<1$.

If we assume that the input voltage $u(t)$ is sinusoidal, in this case, the solution of the nonlinear differential equation is a trigonometric expansion. The solution can be developed until the order $2\left(\mu^{2}\right)$.

$$
x(t)=x_{0}(t)+\mu x_{1}(t)+\mu^{2} x_{2}(t)+\ldots
$$

where $x_{0}(t)$ is the solution of the nonlinear differential equation of the electrodynamic loudspeaker when we neglect the terms with orders higher than zero, $x_{1}(t)$ is the solution of the nonlinear differential equation when we omit the terms with orders higher than one and smaller than one, $x_{2}(t)$ is the solution of the nonlinear differential equation of the electrodynamic loudspeaker when we neglect the terms with orders smaller than two and higher than two. 
It can be noted that some methods like Volterra Series are interesting but do not show what parameters are really nonlinear. The way of solving the nonlinear differential equation is very important but we think that it is not the most important thing for characterizing electrodynamic loudspeakers. The real problem is to know what parameters vary and how. Furthermore, the ranking of these parameters according to the input current or frequency is of great importance. Indeed, we can think that if the structure of an electrodynamic loudspeaker must be improved, we must know all the defects in the motor or the suspensions.

Let us consider now Eq. (12). The denominator in Eq. (12) contains a nonlinear term. Consequently, it is very difficult to solve this nonlinear differential equation with a nonlinear denominator. One possible solution is to approximate this denominator as follows:

$$
\frac{1}{B l(x)}=\tilde{B l}_{0}+\tilde{B l}_{1} x+\tilde{B l}_{2} x^{2}+\ldots
$$

The previous relation is used as a simplification for solving numerically the nonlinear differential equation. Moreover, we can use a classical trigonometric expansion to solve Eq. (12). In short, the solution of the nonlinear differential equation of the electrodynamic loudspeaker is

$$
x(t)=A \cos (w t)+B \sin (w t)+C \cos (2 w t)+D \sin (2 w t)+\ldots
$$

All the terms $A, B, C, D, \ldots$ are found numerically but an analytical solution is possible if the force factor is approximated. Indeed, the terms $\mathrm{A}$ and $\mathrm{B}$ can be found by inserting $A \cos (w t)+B \sin (w t)$ in the equation (12) with an 
excitation $u(t)$ equals to $P \sin (w t)$ where $P$ is an amplitude. The terms $C$ and $D$ can be found by taking the terms with orders higher than one into account, etc...

\subsection{Theoretical results and position of the small signal parameters according to their created distortions}

We present the position of the small signal parameters according to their created distortions. For this purpose, we solve the nonlinear differential equation by using the serial expansion presented in the previous section but we take into account only one variation of a parameter at a time. Figure 9 shows the created distortions by each Thiele and Small parameter. The level of the input voltage is $10 \mathrm{~V}$ and the frequency of the excitation is $50 \mathrm{~Hz}$. We can see that for this electrodynamic loudspeaker, the nonlinear parameter which creates the most important second-harmonic is the inductance Le of the voice coil, and the nonlinear parameter which creates the most important third-harmonic is the equivalent damping parameter $R_{m s}$. However, when the frequency of the excitation increases, the nonlinear parameters which create more distortions are not the same. Figure 10 presents the created distortions by each Thiele and Small parameter when the frequency of the excitation is $150 \mathrm{~Hz}$. As shown in the previous figure, the level of the input voltage is $10 \mathrm{~V}$ and the frequency of the excitation is $150 \mathrm{~Hz}$. We see that the nonlinear parameter which creates the most important second-harmonic is the equivalent mass $M_{m s}$ and the nonlinear parameter which creates the more important third-harmonic is the damping parameter $R_{m s}$. We sum up 


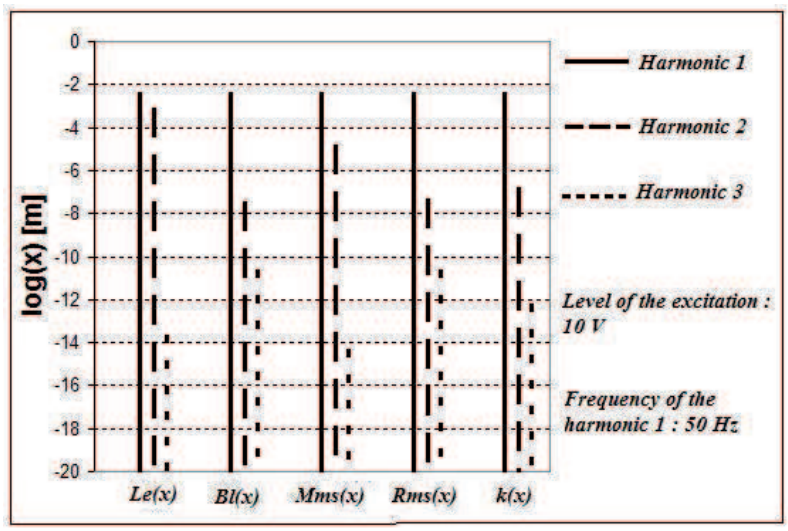

Figure 9: Theoretical spectrums of Thiele and Small parameters: the frequency of the excitation is $50 \mathrm{~Hz}$ and the input voltage is $10 \mathrm{~V}$

\begin{tabular}{||l|c|r||}
\hline Ranking ( 50 Hz) & Parameter(harmonic 2) & $\log [\mathbf{x}]$ \\
\hline 1 & $L_{e}$ & -3.2 \\
\hline 2 & $M_{m s}$ & -4.9 \\
\hline 3 & $R_{m s}$ & -6.8 \\
\hline 4 & $C_{m s}$ & -7.7 \\
\hline 5 & $B l$ & -7.8 \\
\hline
\end{tabular}

Table 2: Ranking of the parameters according to their created distortions( the frequency of the excitation is $50 \mathrm{~Hz}$ )

all the results in tables $2,3,4$ and 5 .

\subsection{Discussion}

The previous section shows that the nonlinear parameters which create the most distortions are not those which are the most nonlinear according to the coil current. For example, figure 7 shows that the equivalent damping parameter $R_{m s}$ is the most nonlinear parameter ( $R_{m s}$ varies a lot with the coil current ) but it is not the parameter which creates the most distortions. On the contrary, the inductance $L_{e}$ of the voice coil varies very little with the coil 


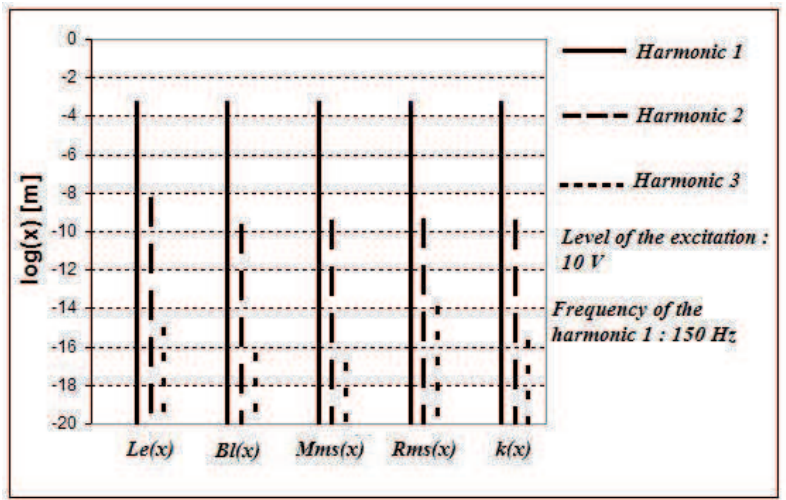

Figure 10: Theoretical spectrums of Thiele and Small parameters: the frequency of the excitation is $150 \mathrm{~Hz}$ and the input voltage is $10 \mathrm{~V}$

\begin{tabular}{||l|c|r||}
\hline Ranking ( 50 Hz) & Parameter(harmonic 3) & $\log [\mathbf{x}]$ \\
\hline 1 & $R_{m s}$ & -11 \\
\hline 2 & $B l$ & -12.5 \\
\hline 3 & $C_{m s}$ & -12.6 \\
\hline 4 & $L_{e}$ & -12.9 \\
\hline 5 & $M_{m s}$ & -14.6 \\
\hline
\end{tabular}

Table 3: Ranking of the parameters according to their created distortions( the frequency of the excitation is $50 \mathrm{~Hz}$ )

\begin{tabular}{||l|c|r||}
\hline Ranking ( 150 Hz) & Parameter(harmonic 2) & $\log [\mathbf{x}]$ \\
\hline 1 & $M_{m s}$ & -5.3 \\
\hline 2 & $L_{e}$ & -6.4 \\
\hline 3 & $R_{m s}$ & -7.7 \\
\hline 4 & $C_{m s}$ & -8.2 \\
\hline 5 & $B l$ & -8.4 \\
\hline
\end{tabular}

Table 4: Ranking of the parameters according to their created distortions( the frequency of the excitation is $150 \mathrm{~Hz}$ ) 


\begin{tabular}{||l|c|r||}
\hline Ranking ( 150 Hz) & Parameter(harmonic 3) & $\log [\mathbf{x}]$ \\
\hline 1 & $R_{m s}$ & -11.7 \\
\hline 2 & $L_{e}$ & -12.6 \\
\hline 3 & $C_{m s}$ & -14.2 \\
\hline 4 & $B l$ & -15 \\
\hline 5 & $M_{m s}$ & -15.1 \\
\hline
\end{tabular}

Table 5: Ranking of the parameters according to their created distortions( the frequency of the excitation is $150 \mathrm{~Hz}$ )

current but at low frequency this parameter creates important distortions.

Moreover, we see that the mechanical compliance $C_{m s}$ is not an important nonlinear parameter since it does not create important distortions. In addition, the ranking of these nonlinearities depend also on input frequency. Such results are interesting for the design of electrodynamic loudspeakers.

\section{BEHAVIOR OF THE CREATED DISTOR- TIONS ACCORDING TO THE VARIA- TIONS OF THE INPUT VOLTAGE}

The previous section presents the ranking of the nonlinear Thiele and Small parameter according to the variation of the frequency excitation. The aim of this section is to show that the distortions created by the nonlinear parameters are more sensitive than the fundamental component according to the variation of the input voltage. To show this, we solve the nonlinear differential equation of the electrodynamic loudspeaker (12). We solve this equation by taking four different amplitudes for the excitation $(1 \mathrm{~V}, 5 \mathrm{~V}, 10 \mathrm{~V}, 50 \mathrm{~V})$ and we represent the spectrum in Fig. 11. The frequency of the excitation 


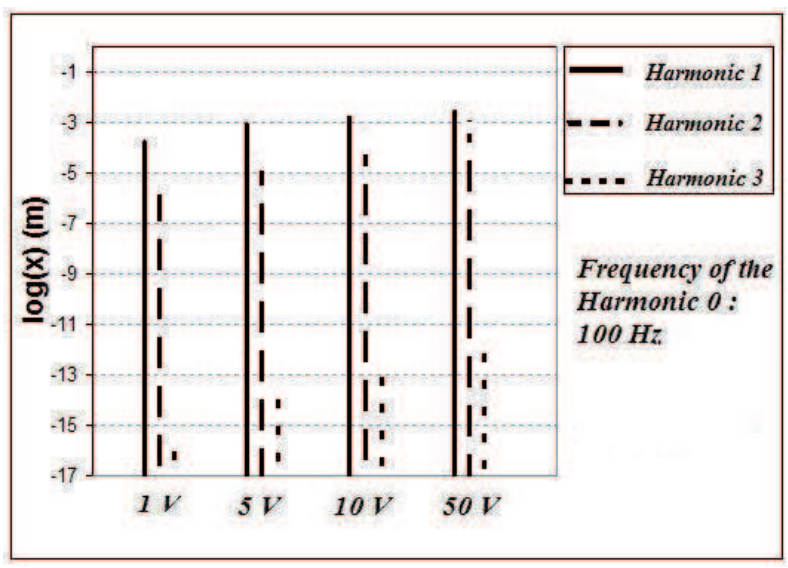

Figure 11: Theoretical spectrum : influence of the input voltage on the created harmonics

\begin{tabular}{||l|c|r|r|r||}
\hline Harmonics & $\mathbf{1 V}$ & $\mathbf{5 V}$ & $\mathbf{1 0 V}$ & \multicolumn{1}{|c|}{$\mathbf{0 V}$} \\
\hline first-harmonic & -3.8 & -3 & -2.8 & -2.7 \\
\hline second-harmonic & -5.9 & -5.6 & -4.2 & -3.4 \\
\hline third-harmonic & -15.8 & -13.5 & -13 & -11.4 \\
\hline
\end{tabular}

Table 6: Ranking of the parameters according to their created distortions (the input frequency is $100 \mathrm{~Hz}$ )

\section{CONCLUSION}

The aim of this paper was the study of the spectrum of the electrodynamic loudspeaker. The experimental method, based on the impedance measurement of an electrodynamic loudspeaker allows us to find all the variations of the Thiele and Small parameters. We can say that this experimental method can be used to characterize many transducers which are described with their electrical impedance. Indeed, an electrical impedance can be seen 
as a nonlinear transfer function which varies with the input current or the input voltage [25]. In section 2 , we have presented a method to derive the coefficients of the nonlinear parameters based on Symplex algorithm. It is noted that a simplifying method has been used in a previous paper [10] and has been improved in this paper.

The small signal coefficients, inserted in the differential equation of the electrodynamic loudspeaker enable us to find the generated harmonics. Many new results are discussed. The equivalent damping parameter is the parameter which is the most nonlinear if we look at its relative variation according to the input current. However, it is not the parameter which creates the most important distortions. This result is important because it gives information about the way of manufacturing an electrodynamic loudspeaker.

More generally, when we take into account the variations of the small signal parameters with the coil current, the mean difference between the experimental and the theoretical values is $0,4 \Omega$, whereas the mean difference is $2,0 \Omega$ with Thiele and Small model with constant parameters.

Another interesting result is the weak variation of the electrical inductance with the input current. It is noted that the electrodynamic loudspeaker characterized in this paper is a good one and is less nonlinear than a bad loudspeaker. However, we see that this weak variation creates important distortions. This is why it can be very important to try to build electrodynamic loudspeakers with constant inductance.

Furthermore, we have seen that these generated harmonics become more and more important when the input voltage increases. This result is in fact 


\section{References}

[1] A. N. Thiele, "Loudspeakers in vented boxes: Part 1 and 2," in Loudspeakers, vol. 1, New York: Audio Eng. Soc., 1978.

[2] W. Klippel, "Dynamic measurement and interpretation of the nonlinear parameters of electrodynamic loudspeakers," J. Audio Eng. Soc., vol. 38, pp. 944-955, 1990.

[3] J. Borwick, Loudspeaker Measurements, in Loudspeaker and Headphone Handbook. Focal Press, oxford ed., 2001.

[4] W. Klippel, "Loudspeaker nonlinearities - cause, parameters, symptoms," J. Audio Eng. Soc., vol. 54, pp. 907-939, 2006.

[5] H. Suzuki and J. Tichy, "Radiation and diffraction effects by convex and concave domes," J. Audio Eng. Soc., vol. 29, pp. 873-881, 1981. 
[6] N. Quaegebeur, A. Chaigne, and G. Lemarquand, "Transient model radiation of asymetric sources: application to loudspeakers," Applied Acoustics, vol. doi:10.1016/japacoust.2009.10.003, 2009.

[7] G. Lemarquand and M. Bruneau, "Large bandwith loudspeaker emitting coherent acoustic waves: nonlinear inter-modulation effects," J. Audio Eng. Soc., vol. 56, pp. 36-44, January 2007.

[8] A. Dobrucki, "Nontypical effects in an electrodynamic loudspeaker with a nonhomogeneous magnetic field in the air gap and nonlinear suspension," J. Audio Eng. Soc., vol. 42, pp. 565-576, 1994.

[9] J. Wright, "An empirical model for loudspeaker motor impedance," J. Audio Eng. Soc., pp. 749-754, October 1990.

[10] R. Ravaud, G. Lemarquand, and T. Roussel, "Time-varying non linear modeling of electrodynamic loudspeakers," Applied Acoustics, vol. 70, pp. 450-458, 2009.

[11] A. J. M. Kaizer, "Modeling of the nonlinear response of an electrodynamic loudspeaker by a volterra series expansion," J. Audio Eng. Soc., vol. 35, pp. 421-433, June 1987.

[12] M. R. Gander, "Moving-coil loudspeaker topology as an indicator of linear excursion capability," J. Audio Eng. Soc., vol. 29, 1981.

[13] M. R. Gander, "Dynamic linearity and power compression in movingcoil loudspeaker," J. Audio Eng. Soc., pp. 627-646, September 1986. 
[14] W. Leach, "Loudspeaker voice-coil inductance losses : Circuit models, parameter estimation and effect on frequency response," J. Audio Eng. Soc., pp. 442-449, 2002.

[15] D. Clark, "Precision measurement of loudspeaker parameters," J. Audio Eng. Soc., pp. 129-141, March 1997.

[16] J. Vanderkooy, "A model of loudspeaker driver impedance incorporating eddy currents in the pole structure," J. Audio Eng. Soc., vol. 37, pp. 119$128,1989$.

[17] G. Lemarquand, "Ironless loudspeakers," IEEE Trans. Magn., vol. 43, no. 8, pp. 3371-3374, 2007.

[18] B. Merit, G. Lemarquand, and V. Lemarquand, "In pursuit of increasingly linear loudspeaker motors," IEEE. Trans. Mag., vol. 45, no. 6, pp. 2867-2870, 2009.

[19] M. Remy, G. Lemarquand, B. Castagnede, and G. Guyader, "Ironless and leakage free voice-coil motor made of bonded magnets," IEEE Trans. Magn., vol. 44, no. 11, 2008.

[20] R. Ravaud and G. Lemarquand, "Modelling an ironless loudspeaker by using three-dimensional analytical approaches," Progress in Electromagnetics Research, PIER 91, pp. 53-68, 2009. 
[21] R. Ravaud and G. Lemarquand, "Design of ironless loudspeakers with ferrofluid seals: analytical study based on the coulombian model," Progress in Electromagnetics Research B, vol. 14, pp. 285-309, 2009.

[22] R. Ravaud, G. Lemarquand, and V. Lemarquand, "Magnetic pressure and shape of ferrofluid seals in cylindrical structures," J. Appl. Phys., vol. 106, no. 3, p. 34911, 2009.

[23] I. E. C, Sound. System. Equipment, Part 5: Loudspeaker. Edition 3.1, 11-09-2007.

[24] J. Dalmont, C. Nederveen, and N. Joly, "Radiation impedance of tubes with different flanges:numerical and experimental investigations," J. of Sound and Vibration, vol. 244, no. 3, pp. 505-534, 2001.

[25] J. C. Gille, P. Decaulne, and M. Pelegrin, Systemes asservis non lineaires. Bordas Editeur, Paris, France, 1981. 